\title{
ADAPTIVE DIVERGENCE IN DARWIN'S RACE: HOW COEVOLUTION CAN GENERATE TRAIT DIVERSITY IN A POLLINATION SYSTEM
}

\author{
Feng Zhang, ${ }^{1}$ Cang Hui, ${ }^{1,2}$ and Anton Pauw ${ }^{3}$ \\ ${ }^{1}$ Centre for Invasion Biology, Department of Botany and Zoology, Stellenbosch University, Private Bag X1, Matieland 7602, \\ South Africa \\ 2E-mail: chui@sun.ac.za \\ ${ }^{3}$ Department of Botany and Zoology, Stellenbosch University, Private Bag X1, Matieland 7602, South Africa
}

Received March 20, 2012

Accepted August 9, 2012

\begin{abstract}
Understanding how reciprocal selection shapes interacting species in Darwin's coevolutionary race is a captivating pursuit in evolutionary ecology. Coevolving traits can potentially display following three patterns: (1) geographical variation in matched traits, (2) bias in trait matching, and (3) bimodal distribution of a trait in certain populations. Based on the framework of adaptive dynamics, we present an evolutionary model for a coevolving pollination system involving the long-proboscid fly (Moegistorhynchus longirostris) and the long-tubed iris (Lapeirousia anceps). The model successfully demonstrates that Darwin's hypothesis can lead to all three patterns if costs are involved. Geographical variation in matched traits could be driven by geographical variation in environmental factors that affect the cost rate of trait escalation. Unequal benefits derived from the interaction by the fly and the flower could potentially cause the bias in trait matching of the system. Different cost rates to trait elongation incurred by the two species and weak assortative interactions in the coevolutionary race can drive divergent selection (i.e., an evolutionary branching) that leads to the bimodal distribution of traits. Overall, the model highlights the importance of assortative interactions and the balance of costs incurred by coevolving species as factors determining the eventual phenotypic outcome of coevolutionary interactions.
\end{abstract}

KEY WORDS: Adaptive dynamics, coevolution, evolutionary trap, geographic mosaic theory, geographical variation, pollination, Red Queen dynamics.

Ever since Darwin $(1859,1862)$, the interaction between longproboscid pollinators and long-tubed flowers has been studied extensively to gain insight into the process of coevolution. Besides evidence of a strong correlation between coevolving traits, three additional patterns have emerged from recent literature: (1) geographical variation in the degree of trait escalation; (2) a bias in trait matching in favor of one species; and (3) the bimodal distribution of traits within some populations. First, although strongly correlated in most populations, the level of trait escalation typically varies considerably across the landscape (Anderson and Johnson 2008; Pauw et al. 2009). Second, coevolving traits often do not match each other perfectly; instead, there is a consistent bias in trait matching, that is, the proboscis is either longer or shorter than the floral tube across all populations (Anderson and Johnson 2008; Pauw et al. 2009). Third, in certain populations the length of floral tubes or proboscises can exhibit a bimodal distribution where "longs" and "shorts" coexist within one population (Wasserthal 1997; Pauw et al. 2009; Anderson et al. 2010). Notably, patterns of geographical variation and bias in trait matching have been widely observed in a range of coevolutionary systems (Steiner and Whitehead 1990; Benkman et al. 2003; Thompson 2005; Toju 2011), yet the pattern of bimodal traits 
in a single population have mainly been reported in pollination systems with elongated coevolving traits (Wasserthal 1997; Pauw et al. 2009; Anderson et al. 2010; but see Duffy et al. 2008). Although controversial hypotheses exist on the evolutionary mechanisms driving trait variation, such as the geographic mosaic hypothesis (Thompson 2005) and trait escalation by coevolution or pollinator shift (i.e., whehter two-side reciprocal selection or oneside selection drives trait escalation in focal systems; Wasserthal 1997, 1998; Nilsson 1998; Whittall and Hodges 2007; Ennos 2008; Hodges and Whittall 2008), a quantitative model that gives rise to these observed patterns is still lacking.

According to Darwin's coevolutionary hypothesis (Darwin 1862; Nilsson 1998), effective feeding occurs when proboscis length exceeds floral tube length, because the pollinator is then able to drain all of the nectar from the flower; in contrast effective pollination occurs when tube length exceeds proboscis length because this ensures sufficient contact with the stigma and anthers near the entrance of the floral tube. Thus, the two traits impose reciprocal directional selection on each other leading to an escalating arms race (Janzen 1980; Benkman et al. 2003; Langmore et al. 2003; Pauw et al. 2009). Indeed, a large number of field studies have shown that proboscis length can act as a selective agent on floral tube length (Herrera 1993; Johnson and Steiner 1997; Nilsson 1998; Maad 2000; Alexandersson and Johnson 2002; Little et al. 2005; Muchhala and Thomson 2009; Navarro and Medel 2009), whereas a recent experiment provides further support for reciprocal selection by showing that the benefits to both plants (pollen grains received) and pollinators (nectar consumed) depend on the relative lengths of their interacting traits (Pauw et al. 2009), with the result that proboscis length and the floral tube length serve as a selective agent on each other.

Darwin's hypothesis and the new data that support it help us to understand how reciprocal selection can lead to trait matching and trait escalation, but do not offer an explanation for the additional patterns of trait variation observed in the field. Furthermore, Darwin's hypothesis does not incorporate the cost to trait escalation, which could serve as a morphological or physiological constraint in this coevolutionary race (Wallace 1867). Because the rate of cost to trait escalation is likely to vary with environmental context, geographical variation in costs could potentially explain the geographical variation in coevolved traits (Steiner and Whitehead 1990; Anderson and Johnson 2008; Pauw et al. 2009). For example, in areas with strong winds, pollinators may experience difficulty in inserting their proboscises into flowers, whereas longtubed flowers may be easily desiccated or damaged (Pauw et al. 2009). The rate of cost to trait escalation could further be species specific, causing imbalanced selection pressure for coevolving species that could potentially lead to complicated coevolutionary trajectories.
Hence, our aim here is to present a model of the coevolutionary interaction between long-proboscid pollinators and longtubed flowers that extends Darwin's model by explicitly incorporating the costs of trait escalation. The model is built on the framework of adaptive dynamics (Dieckmann et al. 2004; Dercole and Rinaldi 2008) and explores how variation in the benefits and costs of trait escalation affect the evolutionary outcome. Important insights are gained by parameterizing the model with observational data on the interaction between the long-proboscid fly Moegistorhynchus longirostris (Nemestrinidae) and the longtubed iris Lapeirousia anceps (Iridaceae) in the Cape Floristic Region of South Africa (Manning and Goldblatt 1997). Surprisingly, this simple model with few parameters is able to produce the observed range of phenotypic outcomes, that is, geographical variation, bias in trait matching, and the bimodal distribution of traits. The model indicates new avenues for empirical studies aimed at testing Darwin's coevolutionary hypothesis and other contending theories about the evolutionary diversification of traits, and is applicable to a range of coevolutionary systems (both mutualistic and antagonistic) that involve explicit benefits and costs.

\section{Materials and Methods STUDY SPECIES AND DATA}

The coevolution of the long-tubed iris (L. anceps) and the longproboscid fly (M. longirostris) in the lowlands along the west coast of the Cape Floristic Region, South Africa, was chosen as the study system (Fig. 1). The study area covered eight sites of typical fynbos vegetation, with nutrient-poor, sandy soil under Mediterranean climate. In all eight sites, $L$. anceps is the dominant flowering plant and $M$. longirostris is the only pollinator for L. anceps (Pauw et al. 2009). Other long-tubed plant species do exist at the study sites and are visited by $M$. longirostris, but are rarer and thus less important sources of nectar. The lengths of fly proboscis and floral tube were randomly sampled within each site. At one site, 81 pollination events were recorded in October 2006, with the percentage of available nectar consumed by pollinators and the number of pollen grains received by floral stigmas recorded, and the lengths of the interacting fly proboscis and floral tube measured (see Pauw et al. 2009 for details). The volume of nectar consumed was calculated from the difference in nectar level inside the floral tube before and after the pollinator visit. The number of pollen grains received was determined by removing the stigmas after the pollinator visit and counting the number of adhered pollen grains under a microscope. Although the selection study was conducted in only one population, the 81 interactions spanned the range trait matching observed across all populations: floral tube longer than proboscis; floral tube equal to proboscis; floral tube shorter than proboscis. 


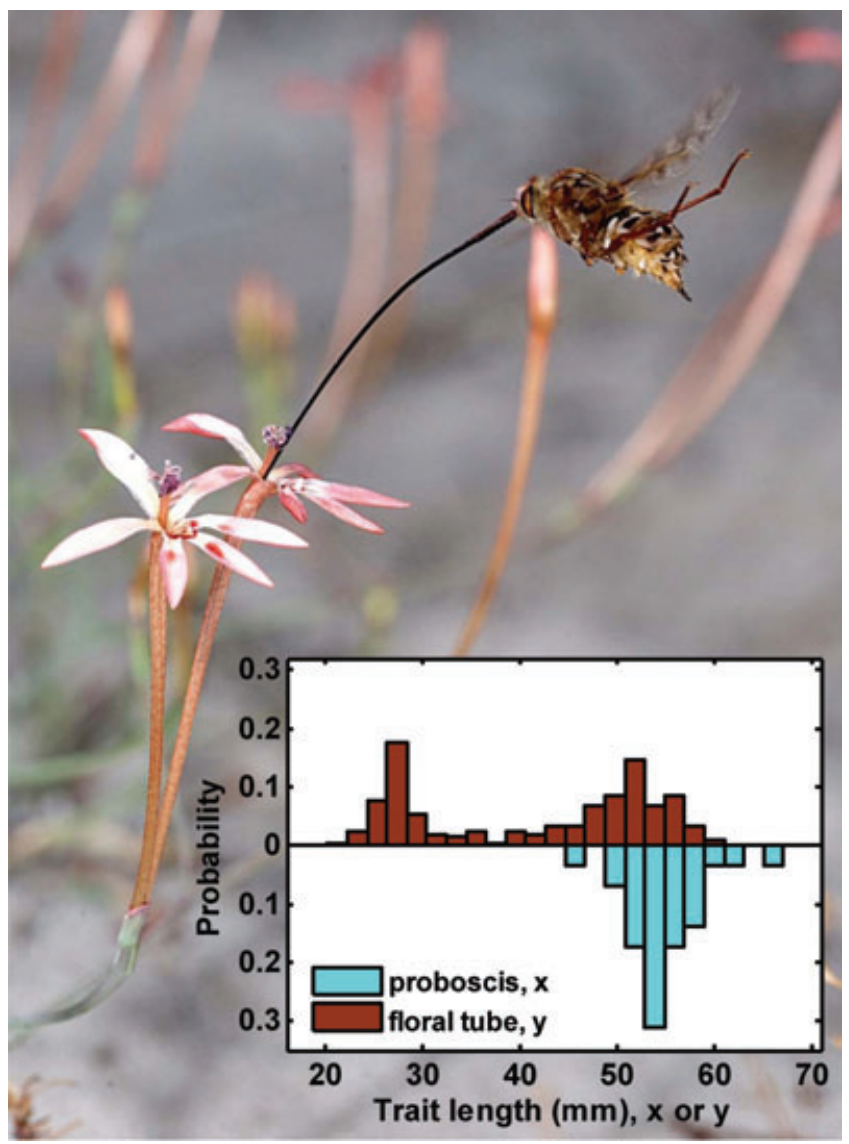

Figure 1. Coevolution is exemplified by the interaction between the long-proboscid fly, Moegistorhynchus longirostris, and the long-tubed iris, Lapeirousia anceps, in South Africa. The relative length of proboscis and floral tube determines the fitness benefits to fly and flower in a race for elongation. Inset indicates the coevolutionary community in Mamre site (Pauw et al. 2009), including the bimodal frequency distribution of floral tube lengths and the unimodal distribution of fly proboscis lengths.

\section{COEVOLUTIONARY MODEL}

Following Darwin's coevolutionary hypothesis, the individual benefits of both species were dependent on the relative length of their interacting traits. For example, when the proboscis becomes longer in relation to the floral tube, the benefit to the pollinator increases, whereas the benefit to the flower declines (Pauw et al. 2009). In the mutualistic interactions, the benefit to a pollinator was quantified by the proportion of nectar consumed per visit (assuming the independence of nectar quantity to floral specifics), and the benefit to a flowering plant by the probability of successful pollination. Let $x$ and $y$ denote the lengths of fly proboscis and floral tube, respectively. A logistic equation was fitted to the data for formulating the benefits (Fig. 2):

$$
b_{i}(x, y)=\frac{1}{1+\exp \left[(-1)^{i} k_{i}(x-y)+w_{i}\right]},
$$

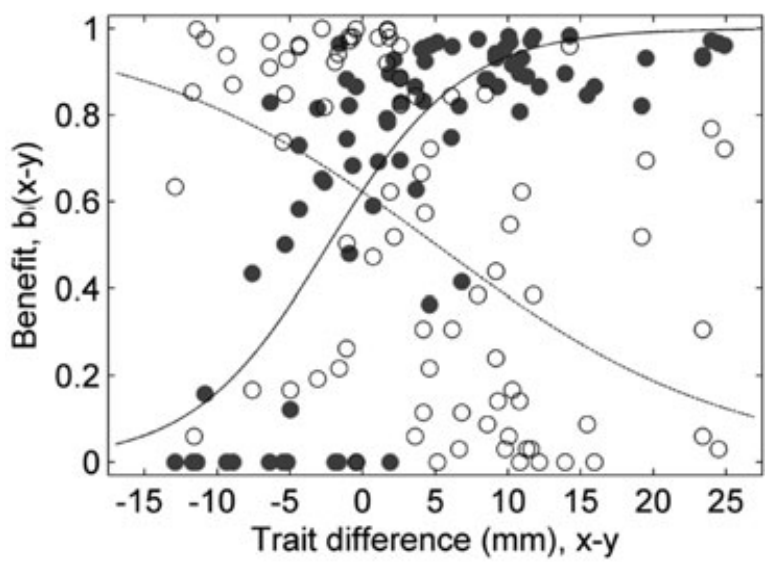

Figure 2. The fitness benefits to pollinators (fraction of nectar consumed; black dots) and plants (probability of successful pollination; open dots) in the interaction between the long-proboscid fly, Moegistorhynchus longirostris, and the long-tubed iris, Lapeirousia anceps. Lines indicate the nonlinear regression curves for the pollinators ( $i=1$ in eq. 1 ; solid line) and for the plants ( $i=2$ in eq. 1; dashed line).

where $i$ indicates either the pollinator $(i=1)$ or the flower $(i=2), k_{i}(>0)$ the sensitivity of the benefit to the relative difference of the two traits $(x-y)$, and $w_{i}$ a parameter in the logistic equation indicating the trait difference $(x-y)$ when the benefit equals $1 / 2$. We regard the benefit as a proxy of fitness, that is, an implicit assumption is that the benefit gained during the interaction is positively correlated with the number of offspring produced. Similar fitness proxies have been used in studies of coevolution between parasites and host plants (Bergelson et al. 2001; Toju and Sota 2006), between toxic newts and garter snake predators (Brodie and Ridenhour 2003), and between the length of nectar spur in plants and the proboscis length of pollinators (Nuismer et al. 2010).

Furthermore, because the elongation of these two traits inevitably incurs an increasing cost, we assume that the cost function follows an exponential form, meaning that the change rate of the cost with the trait elongation is constant (i.e., a Malthusian rate),

$$
c_{i}(s)=\exp \left(\lambda_{i} s\right)-1
$$

where $s$ indicates the length of the trait, $\lambda_{i}$ the increasing cost per unit length of elongation (hereafter, cost rate), and the term " -1 " to ensure that the minimum length has negligible cost, $c_{i}(0)=$ 0 . The exact form of the cost is likely to be determined by the morphology and physiology of the species, and can be further affected by the abiotic environment of the site (e.g., high wind velocity; Anderson and Johnson 2008; Pauw et al. 2009); that is, the cost rate varies among geographic sites due to the environmental variation. An additional analysis suggests that other forms of cost functions do not affect the results substantially (see Appendix S1). 

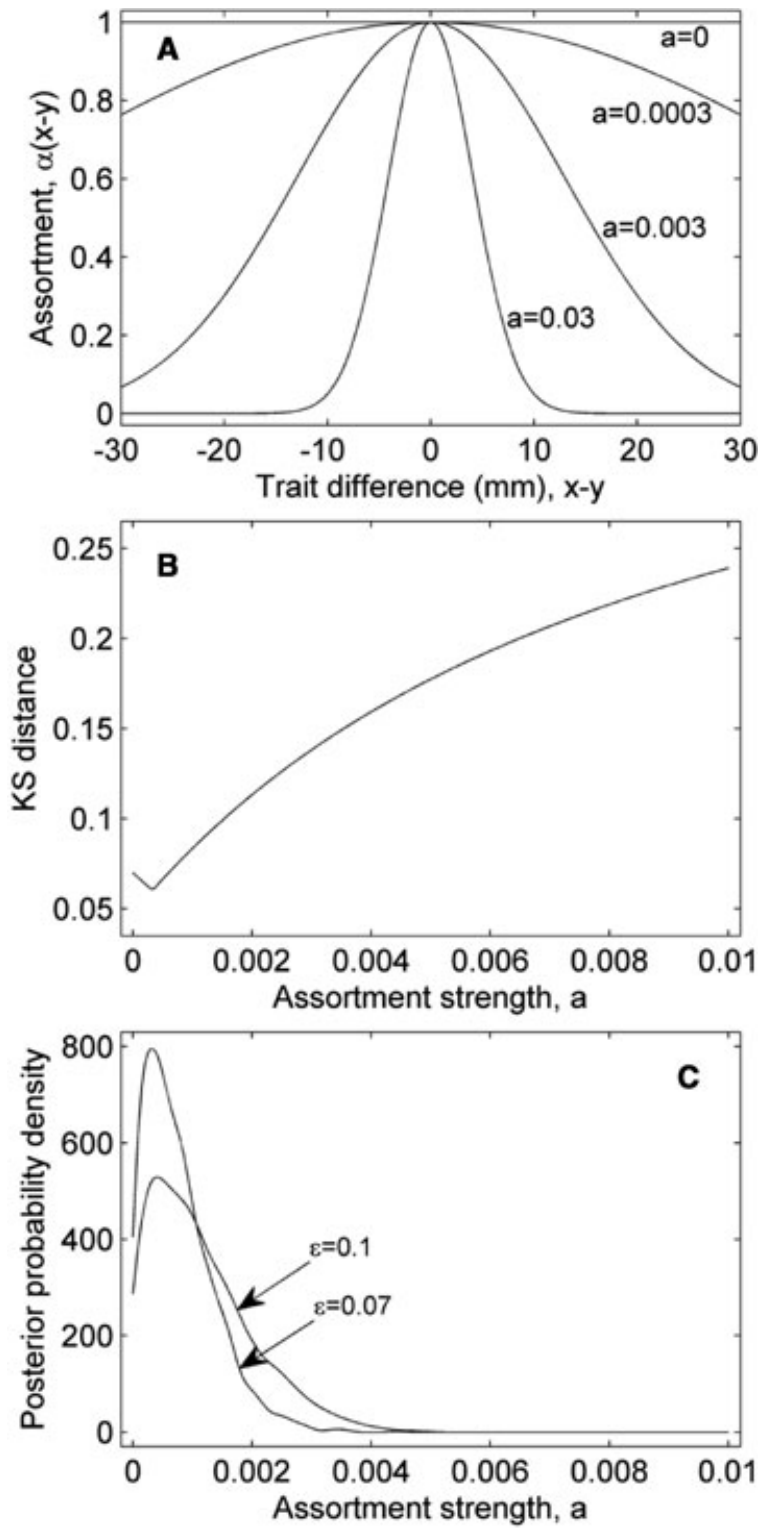

Figure 3. Assortment of pollinators for flowers with tube lengths that match proboscis length. (A) The assortment as a function of trait difference (eq. 3), where parameter $a(>0)$ indicates the strength of assortment; (B) the relationship between the assortment strength and the Kolmogorov-Smirnov (KS) distance in the Moegistorhynchus longirostris- Lapeirousia anceps system (details see section Parameterization); (C) the posterior probability densities of the assortment strength resulting from approximate Bayesian computation at two different tolerant levels $(\varepsilon)$ (details see section Parameterization). The curves are estimated from $\mathbf{2 0 0 0}$ random draws of accepted values of parameter a using approximation Bayesian computation.

We further assume that a pollinator with a certain proboscis length $(x)$ assortatively visits flowers of a similar tube length $(y)$. This assortment determines the possibility that the pollinator and flower interact with each other when encountered and has been represented by a Gaussian function (Kiester et al. 1984; Fig. 3A):

$$
\alpha(x, y)=\exp \left[-a(x-y)^{2}\right]
$$

where $a(>0)$ indicates the strength of assortment (i.e., the tolerance level of a pollinator to flowers of different tube lengths; specifically $a=0$ means a pollinator visits flowers randomly without assortment; Fig. 3A). An alternative logistic function of the assortment, depicting that the flies are likely to visit any flowers with shorter floral tubes than their proboscis, regressed the data worse than the Gaussian function (see Appendix S2) and was thus not presented here. Consequently, the probability that an $x$-proboscis-length pollinator and a $y$-tube-length flower will interact with each other should depend on both the assortment and the probability (frequency) distribution of traits in the population, leading to the following bivariate distribution of traits among interacting plants and pollinators (i.e., the probability for specific pair of traits to interact in the population; Doebeli and Dieckmann 2000):

$$
\varphi(x, y)=\frac{p(x) q(y) \alpha(x, y)}{\iint p(u) q(v) \alpha(u, v) d u d v},
$$

where $p(x)$ and $q(y)$ are the probability distributions of trait $x$ and $y$, respectively. Therefore, the average per capita benefits gained by a pollinator with an $x$-length proboscis and by a flower with a $y$-length tube can thus be calculated by $\int b_{1}(x, y) \varphi(x, y) d y / p(x)$ and $\int b_{2}(x, y) \varphi(x, y) d x / q(y)$, respectively; here the integral gives the total benefit gained by a specific trait in the population.

If there is a pollinator mutant $\left(x^{\prime}\right)$, or a flower mutant $\left(y^{\prime}\right)$, in a pure-line population $(p(x)=q(y)=1)$, the fitness of these mutants can be estimated approximately by the average benefit minus cost:

$$
\begin{aligned}
& f_{1}\left(x^{\prime}, x, y\right)=b_{1}\left(x^{\prime}, y\right) \frac{\alpha\left(x^{\prime}, y\right)}{\alpha(x, y)}-c_{1}\left(x^{\prime}\right) \\
& f_{2}\left(y^{\prime}, x, y\right)=b_{2}\left(x, y^{\prime}\right) \frac{\alpha\left(x, y^{\prime}\right)}{\alpha(x, y)}-c_{2}\left(y^{\prime}\right)
\end{aligned} .
$$

If the fitness of the mutant is greater than the average fitness of the resident population (e.g., $f_{1}\left(x^{\prime}, x, y\right)>b_{1}(x, y)-c_{1}(x)$ for flies), the mutant can then invade and replace the resident population; otherwise the mutant dies out. Our model follows the theory of adaptive dynamics, which is a powerful method for studying the evolutionary change of quantitative traits where the fitness can be either frequency or density dependent (Dieckmann and Law 1996; Geritz et al. 1998; Doebeli and Dieckmann 2000; Waxman and Gavrilets 2005). The adaptive dynamics approach assumes that (1) the mutation rates are low so that the population is at the ecological equilibrium when the mutation occurs, (2) the morphological changes are gradual (i.e., small differences between $x^{\prime}$ and $x$, and between $y^{\prime}$ and $y$ ), and (3) the focal organisms are typically asexual. However, the dynamic behavior of this model is mathematically equivalent to the dynamics of a sexual population as long as no evolutionary branching occurs 
(Waxman and Gavrilets 2005). Dynamics beyond the branching point needs further consideration of assortative mating (see below for details).

The adaptive evolutionary dynamics of proboscis and floral tube length can be determined by the derivatives of the mutant fitness: $\partial f_{1}\left(x^{\prime}, x, y\right) /\left.\partial x^{\prime}\right|_{x^{\prime}=x}$ and $\partial f_{2}\left(y^{\prime}, x, y\right) /\left.\partial y^{\prime}\right|_{y^{\prime}=y}$ (see Appendix S3). If the derivative is positive, the trait will escalate (longer traits invade the population); otherwise, the trait length will decline (shorter traits invade the populations). The evolutionary equilibrium, also known as the evolutionary singular point $\left(x^{*}, y^{*}\right)$, can be derived by setting these two derivatives to zero (Dieckmann and Law 1996; Geritz et al. 1998). The equilibrium becomes an evolutionary attractor (i.e., the trait length gradually converges to the equilibrium) if and only if the Jacobian matrix of the evolutionary dynamics at the singular point has eigenvalues with negative real parts (see Appendix S3). When the evolutionary singular point is unstable, the periodical change of trait lengths could potentially lead to an evolutionary cycling, representing the Red Queen dynamics (Dieckmann et al. 1995). It is possible for an attractor to represent a minimum fitness for either or both traits provided that one or both of the following conditions are satisfied:

$$
\begin{aligned}
& \left.\frac{\partial^{2} f_{1}\left(x^{\prime}, x^{*}, y^{*}\right)}{\partial x^{\prime 2}}\right|_{x^{\prime}=x^{*}}>0, \\
& \left.\frac{\partial^{2} f_{2}\left(y^{\prime}, x^{*}, y^{*}\right)}{\partial y^{\prime 2}}\right|_{y^{\prime}=y^{*}}>0 .
\end{aligned}
$$

This will potentially lead to an evolutionary branching (i.e., the rise of polymorphism) for one or both species and thus a bimodal frequency distribution of the branched trait within the population (Dieckmann and Law 1996; Doebeli and Dieckmann 2000). For example, if the inequality (7) holds, the trait $y^{*}$ stands for a minimum fitness where traits either longer or shorter than $y^{*}$ can then invade this resident population. However, as shown in the Results, this is not necessarily true for coevolutionary populations, for example, even if the inequality (7) holds, individuals with the two divergent floral traits might still not be able to coexist (no evolutionary branching occurs), forcing the population into the minimum fitness, that is, an evolutionary trap.

\section{PARAMETERIZATION}

To estimate the model parameters, a nonlinear regression was used to fit the logistic curve in equation (1) to the percentage of nectar consumed and the probability of successful pollination in relation to the trait difference $(x-y)$. The original records regarding the number of pollen grains gained by floral stigmas was converted to the probability of successful pollination according to $1-$ $(1-p)^{n}$, where $n$ represents the number of received pollen grains and $p$ the probability of successful pollination by a single pollen grain. Although no studies have examined the relationship between seed productivity and the number of pollen grains received for this species, preliminary analysis suggests that the model outcomes were unchanged qualitatively with respect to parameter $p$ (see Appendix S4), and thus we choose a low value $(p=0.03)$ for the demonstration.

To quantify the evidence for assortment in the interactions between the fly and flower, we regressed the potential probability distributions (eq. 4) of the trait difference $(x-y)$ for interacting individuals at different levels of assortment strength (parameter $a$ in eq. 3) with records from the 81 pairs of pollination events. For each value of parameter $a$, we calculated the KolmogorovSmirnov (KS) distance between the probability distribution and the empirical distribution of real observations, whereby the value of parameter $a$ with the smallest KS distance can be considered a maximum likelihood estimate of the real assortment strength. To double check, approximate Bayesian computation (Beaumont 2010) was also used to estimate the assortment strength (parameter $a$ ). Specifically, for a given value of parameter $a$, we randomly generated 81 pairs of interactions according to the corresponding probability distribution of trait difference (eq. 4). If the KS distance between the random draws and real observations is less than a tolerant level $\varepsilon$, we accept the given value as a possible value for parameter $a$; otherwise, we reject it. Using kernel density method (MATLAB v7.10.0, The MathWorks Inc., Natick, Massachusetts, USA), we estimated the posterior probability density of the assortment strength for 2000 given values of parameter $a$ and then calculated the estimate and its confidence interval from the posterior probability density.

To assess the effect of variation in the cost rate of trait escalation and variation in the strength of assortative interaction on the coevolutionary dynamics, we ran a sensitivity test and numerically calculated the solutions to this model (i.e., the coevolutionary outcomes) for 160,000 combinations of parameters, representing 200 values for each cost rate $\left(\lambda_{1}\right.$ and $\lambda_{2}$ in eq. 2$)$ and four levels of assortment strength $(a)$, with other parameters estimated from real data. These solutions allowed us to explore the full-range of coevolutionary behaviors in the coevolutionary interaction between flies and flowers, and allowed comparison of model predictions (as evolutionarily stable attractors) with observations at different geographic localities. Moreover, similar sensitivity tests of the parameters in the benefit function (Appendix S5) and in other forms of the cost function (Appendix S1) were also performed to ensure the robustness of the results.

\section{SIMULATION}

Predictions from the model, specifically regarding the condition for evolutionary branching and evolutionary cycling (see Results), were further verified via an individual-based simulation, representing the coevolution of two populations with a fixed number 
of individuals (2000 individuals per species) and discrete generations. The fitness of each fly with an $x$-length proboscis and of each flower with a $y$-length tube was measured by the expected benefit minus cost:

$$
\begin{aligned}
& f_{1}(x)=\frac{1}{p(x)} \sum_{y} b_{1}(x, y) \varphi(x, y)-c_{1}(x) \\
& f_{2}(y)=\frac{1}{q(y)} \sum_{x} b_{2}(x, y) \varphi(x, y)-c_{2}(y)
\end{aligned}
$$

Individuals of the next generation were randomly sampled according to the probability distribution of traits weighted by their fitness in the parental populations. Individuals inherited their parental traits except for mutants (at a mutation rate of $\mu=0.01$ ), and the trait of a mutant was drawn from a Gaussian distribution with the parental trait as the mean and a variance of $\sigma^{2}=0.25$. The mutation rate $(\mu)$ and trait variance $\left(\sigma^{2}\right)$ only affect the evolutionary speed (see eq. S2 in Appendix S3 and also Appendix S6), and therefore we assigned low values to both parameters according to the assumptions of adaptive dynamics (i.e., low mutation rate and gradual morphological changes). Although asexual reproduction was implicitly assumed in the simulation, the evolutionary behavior is similar to sexual population before the evolutionary branching (Kisdi and Geritz 1999; Waxman and Gavrilets 2005), whereas the event of evolutionary branching was also simulated for populations with sexual reproduction and assortative mating (see Appendix S7; Dieckmann and Doebeli 1999; Doebeli and Dieckmann 2000).

\section{Results}

\section{PATTERNS AND PARAMETERIZATION}

In the eight sites studied, there were five sites where the lengths of fly proboscis and floral tube matched with each other, two sites mismatched, and one site had a bimodal distribution of floral tube length (inset of Fig. 1; Appendix S8). Matched traits varied significantly among sites in the level of escalation, and across most populations the mean proboscis lengths exceeded the mean floral tube lengths (Appendix S8). Notably, for the population with a bimodal distribution of floral tubes, the fly proboscis matched with the long floral tubes, whereas the short floral tube was the shortest among all populations across eight sites, suggesting that it is impossible to explain the existence of these short-tube individuals simply by trait remixing (i.e., gene flow) from among the sampled populations (inset of Fig. 1; Appendix S8).

The forms of model parameters (eqs. 1 and 3 ) were supported by the experimental observations. The logistic equations (eq. 1) fitted well to the benefit relative to trait difference $(x-y)$ (Fig. 2), with $k_{1}=0.2155 \pm 0.0353$ (mean $\pm \mathrm{SE}, P<0.01$ ) and $w_{1}=-0.5094 \pm 0.1557(P<0.01)$ for the percentage of nectar consumed by pollinators $\left(R^{2}=0.77\right)$ and $k_{2}=0.0983 \pm 0.0251$
$(P<0.01)$ and $w_{2}=-0.4980 \pm 0.1998(P<0.01)$ for the probability of successful pollination $\left(R^{2}=0.45\right)$.

The minimum KS distance between the probability distribution of trait difference $(x-y)$ for interacting individuals (eq. 4) with assortative interactions (eq. 3 ) and the real distribution of the observations was reached when the assortment strength $a$ equals 0.000345 (Fig. 3B). The posterior probability density estimated from the approximate Bayesian computation also reached the peak at the same point, with the $95 \%$ confidence interval being $[0,0.0026]$ under the tolerance $\varepsilon=0.1$ and $[0,0.0018]$ under the tolerance $\varepsilon=0.07$ (Fig. 3C). This suggests that the real interaction between the fly and flower represents a weak assortative interaction ( $a=0.000345)$. We, however, cannot statistically reject the possibility of a completely random interaction (i.e., without assortment) because $a=0$ is included in the $95 \%$ confidence interval. Therefore, we reported the model predictions under both scenarios (i.e., no and weak assortative interactions). In addition, it is important to realize the experiment was not set up with the intention of testing assortment, and a specifically designed test will be conducted as part of a future study.

\section{COEVOLUTIONARY DYNAMICS}

Without assortative interactions, the system can have either one or two positive evolutionary equilibria (Fig. 4A). If the system has one equilibrium, it represents one of three potential evolutionary outcomes depending on the cost rates of the two traits: (1) an evolutionarily stable attractor when both inequalities (6) and (7) are violated; (2) an evolutionary trap (i.e., converging to a minimum fitness) for the fly or flower (without entailing an evolutionary branching) when one of the inequalities (6) or (7) is satisfied (Fig. 5A); (3) the dynamics of Red Queen I where the length of proboscis periodically follows the length of floral tube (i.e., a clockwise limit cycle on $x-y$ phase plane; Fig. 5C), or the dynamics of Red Queen II where the length of floral tube periodically follows the length of fly proboscis (i.e., an anticlockwise limit cycle on $x-y$ phase plane; Fig. 5D), if the equilibrium is unstable. Overall, a relatively symmetric (equal) cost rate to trait elongation for the two species resulted in an evolutionarily stable trait length, whereas asymmetric cost rates could push the species that bears comparably higher cost into an evolutionary trap (Fig. 5A) or even trigger Red Queen dynamics (Fig. 5C and D). When the system without assortative interactions had two equilibria (black region in Fig. 4A), the evolutionary outcomes depended on the initial state (Appendix S9). Therefore, the system seldom reached an evolutionarily stable state (attractor) if the cost was low relative to the benefit of elongating the trait (bottom-left corner in Fig. 4A).

Even weakly assortative interactions dramatically changed the evolutionary outcomes of the system (Fig. 4B-D). Interestingly, even with extremely weak assortment for trait matching (e.g., $a=0.0003$ ) species that fell into the evolutionary trap due 


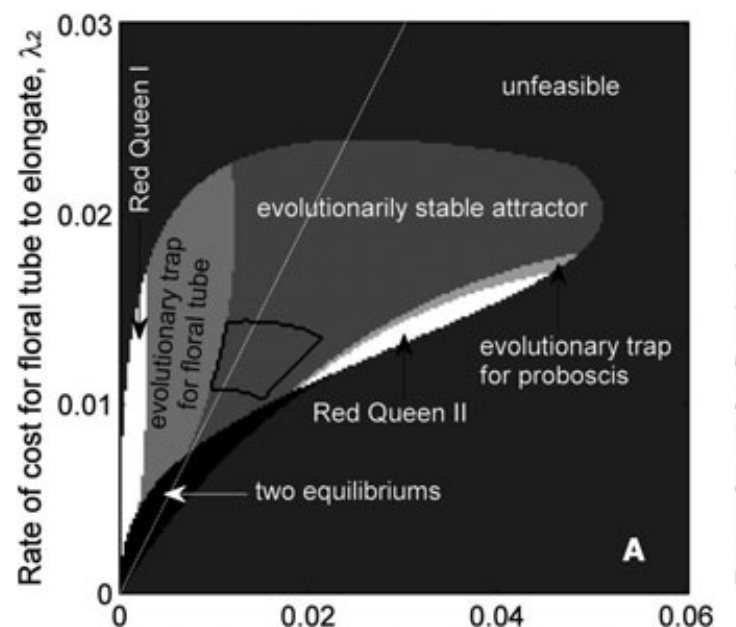

Rate of cost for proboscis to elongate, $\lambda_{1}$

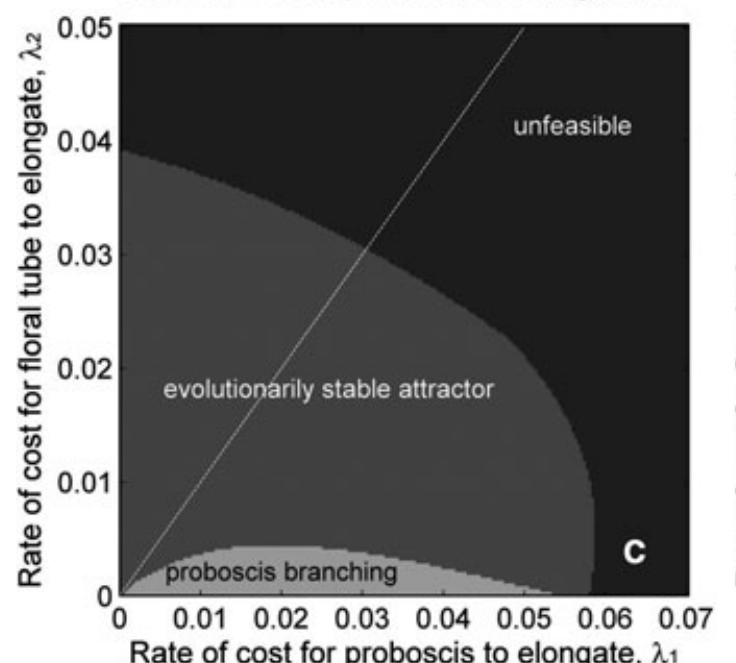

Rate of cost for proboscis to elongate, $\lambda_{1}$

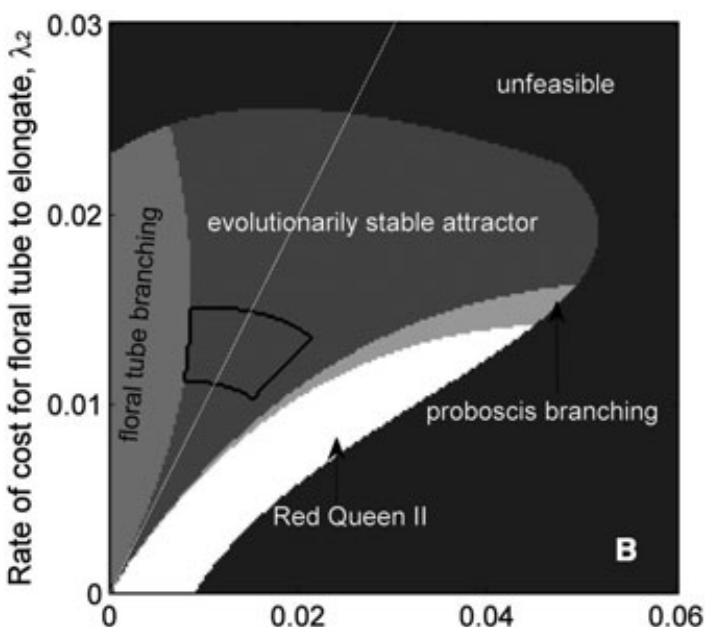

Rate of cost for proboscis to elongate, $\lambda_{1}$

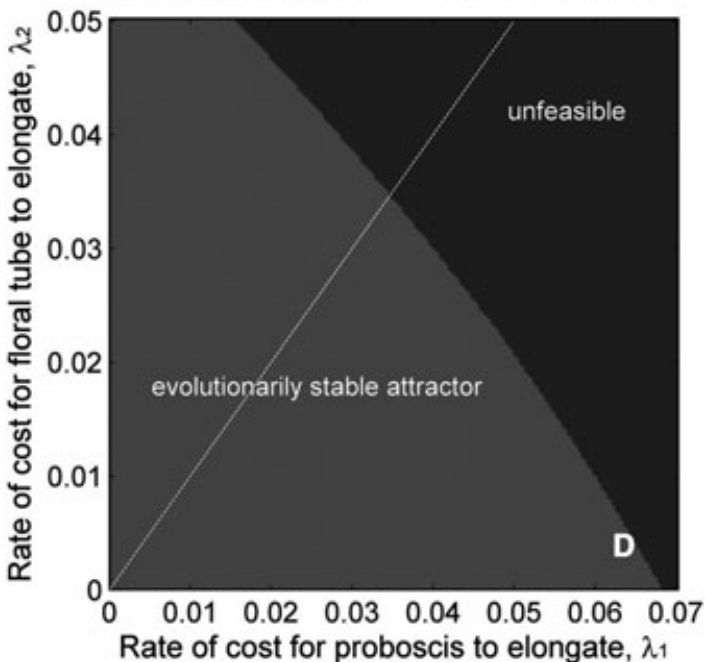

Figure 4. The effects of cost rates $\left(\lambda_{1}\right.$ and $\lambda_{2}$ in eq. 2$)$ on the coevolutionary outcomes in a pollination system with (A) no $(a=0)$, (B) weak $(a=0.0003)$, and strong assortment $(a=0.003$ for $[C]$ and $a=0.03$ for $[D])$. Each plot represents the numerical solutions of $200 x$ 200 combinations of $\lambda_{1}$ and $\lambda_{2}$, with parameters $k_{1}, w_{1}, k_{2}$, and $w_{2}$ assigned from the nonlinear regression (eq. 1; Fig. 2). White straight lines indicate $\lambda_{1}=\lambda_{2}$. Closed black lines in (A) and (B) indicate predictions for the observed real system (the average length of proboscis and floral tube at evolutionarily stable attractors is between $\mathbf{4 0}$ and $80 \mathrm{~mm}$; proboscises are longer than floral tubes), corresponding to the dark gray zone in Figure 6.

to higher cost broke the trap of minimum fitness by an evolutionary branching. For example, if the plant had a much higher cost to elongate its floral tube than the pollinator, both strategies of either reducing cost by shortening the tube or enhancing benefit by elongating it to increase pollen gain became evolutionarily favored. An evolutionary branching occurred and a bimodal distribution of floral tube length resulted (Fig. 5B). Noticeably, the two species cannot both experience evolutionary branching in a system with assortative interactions (Fig. 4B-D); similarly without assortative interactions they cannot both experience evolutionary traps (Fig. 4A).

Furthermore, the scenarios of two equilibria and Red Queen I dynamics disappeared even when interactions were weakly assortative (e.g., $a=0.0003$; Fig. 4B). With a further increase in assortment strength, Red Queen II dynamics and evolutionary branching of floral tube also became impossible (e.g., $a=0.003$; Fig. 4C); when the assortment strength was strong, the system can only experience an evolutionarily stable attractor (e.g., $a=0.03$; Fig. 4D). Clearly, assortative interaction is a stabilizing force in the coevolutionary system, and it is thus more difficult for species with strong assortative interaction than those with weak assortative interaction to exhibit polymorphism in a pollination system.

\section{MODEL PREDICTIONS}

Even though the lengths of proboscis and floral tube did correlate with each other when they were at the evolutionarily stable attractors (Fig. 4), the bias in trait matching was still noticeable (Fig. 6). Models with no or weak assortative interactions but with realistic parameter estimates, successfully predicted the range and direction of the bias in the matching traits (dark gray belt in Fig. 6). 

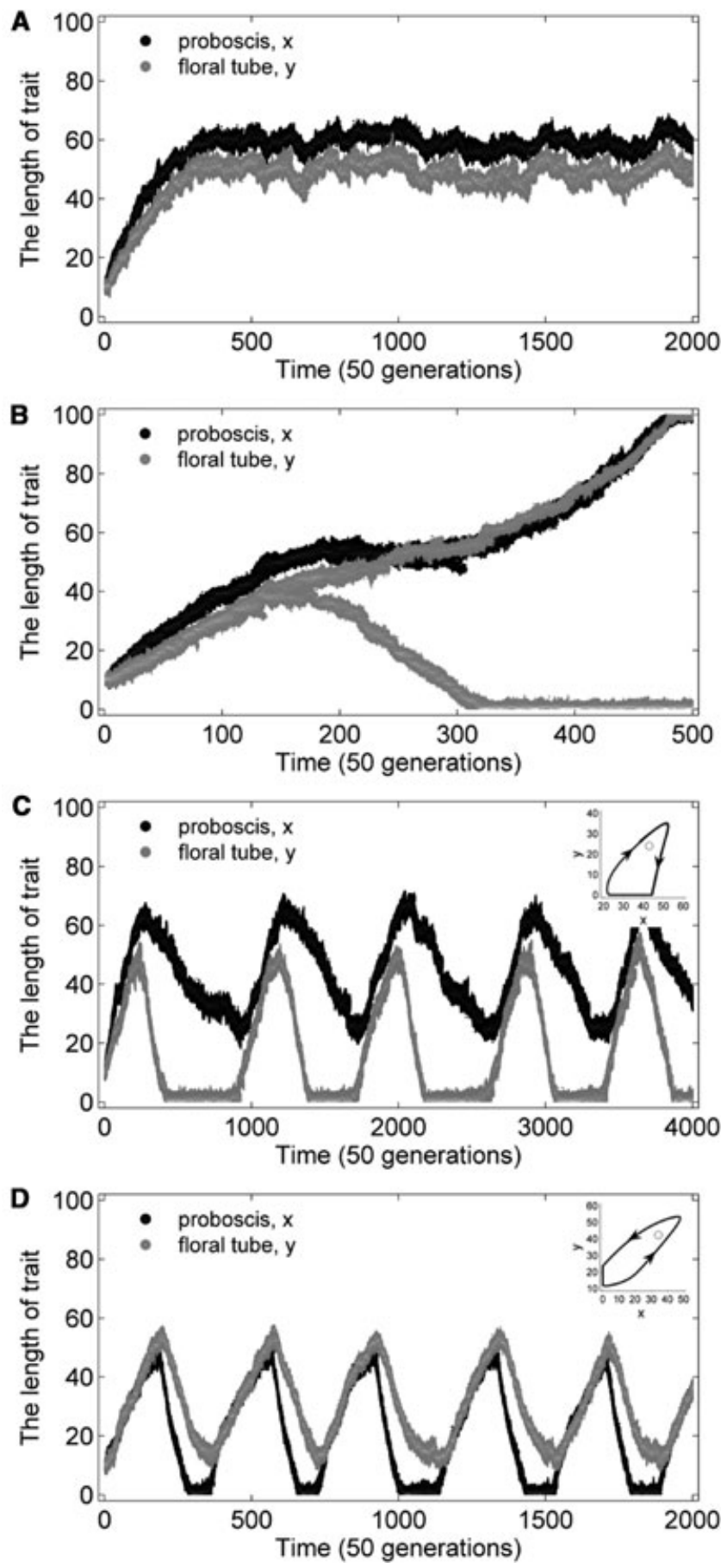

Figure 5. Individual-based simulations of the coevolution between fly proboscis and floral tube. (A) The dynamics of nonassortative interactions ( $a=0$, i.e., completely random interaction between flies and flowers), noting that evolutionary branching does not occur (although the inequality (7) is satisfied); (B) the dynamics of weakly assortative interactions $(a=0.0003)$, with the evolutionary branching as predicted; (C) Red Queen I dynamics; and (D) Red Queen II dynamics. Parameter values are $\lambda_{1}=\mathbf{0 . 0 0 8 5}$ and $\lambda_{2}=0.0125$ for (A); $\lambda_{1}=0.0022$ and $\lambda_{2}=0.0135$ for (B); $\lambda_{1}=$ $0.002, \lambda_{2}=0.012$, and $a=0$ for (C); $\lambda_{1}=0.02, \lambda_{2}=0.009$, and $a=$ 0.0003 for (D). Other parameters $\left(k_{1}, w_{1}, k_{2}\right.$, and $\left.w_{2}\right)$ are set from the nonlinear regression (eq. 1; Fig. 2).
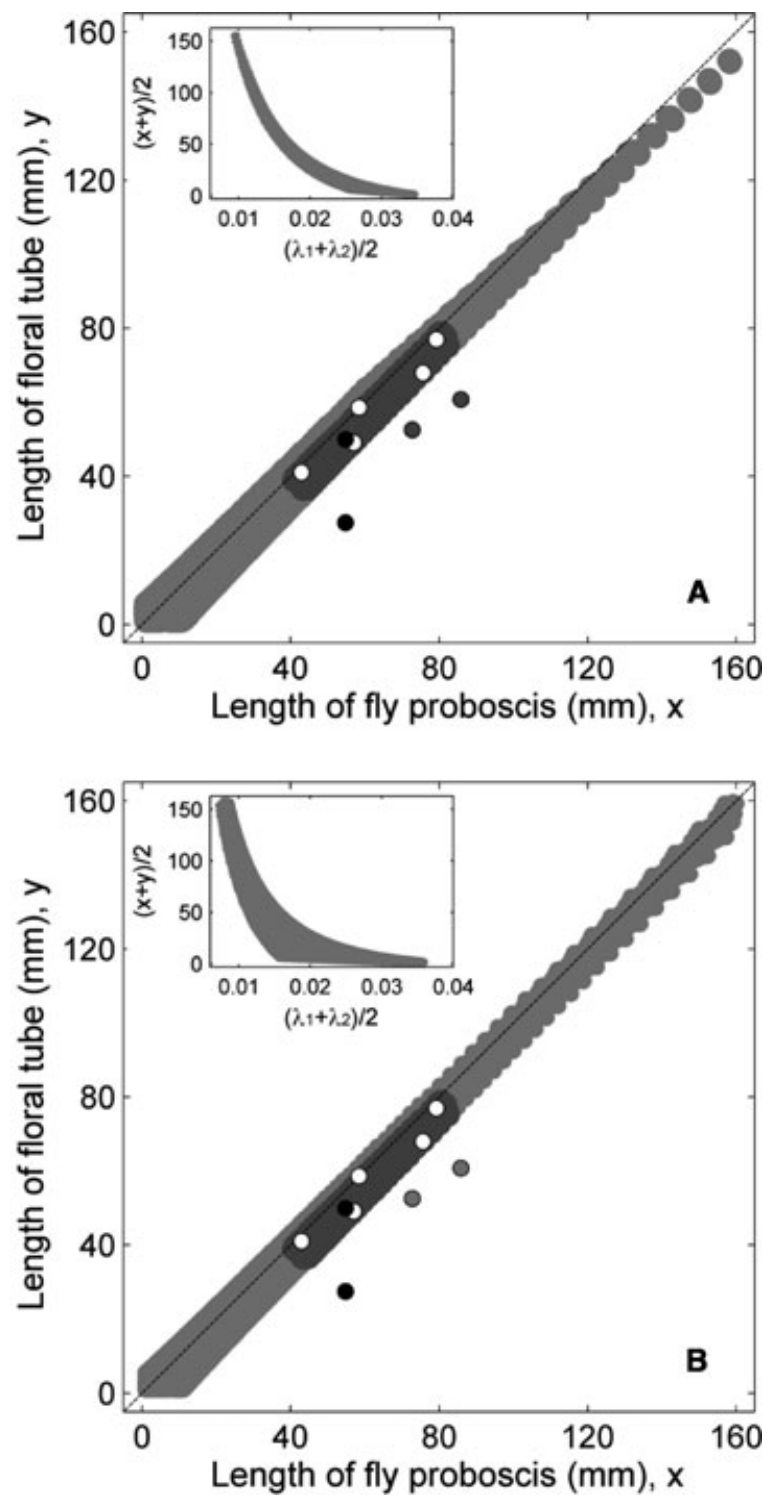

Figure 6. Variation and bias of trait matching in the pollination system with no assortment (A) and weak assortment (B). Gray belts in (A) and (B) are formed by the evolutionary stable attractors of various cost rates $\left(\lambda_{1}\right.$ and $\lambda_{2}$ in eq. 2$)$ in Fig. $4 A$ and $B$, respectively. Dark gray regions are the prediction for the observed populations, corresponding to the combinations of cost functions (points enclosed within the black lines of Fig. 4). Dots indicate the average trait lengths of eight observed fly-flower populations, with one having a bimodal trait distribution (black dots, see also Fig. 1) and two populations potentially representing coevolutionary cold spots (gray dots; Pauw et al. 2009) or snapshots from the Red Queen dynamics (Fig. 5C-D). The insets indicate the relationship between trait length and cost rates.

The three observed cases that lay outside the dark gray belt in Figure 6 represent one shorter floral tube with a bimodal frequency distribution of floral tubes (the longer floral tube lies within the dark gray belt, solid dots) and two other populations with much shorter floral tubes than the proboscises (gray dots). The bimodal 
population could indicate an evolutionary branching (Fig. 5B) whereas the other two mismatched populations could represent a snapshot of Red Queen I dynamics (Fig. 5C). The bias in trait matching could be caused by the asymmetric benefits of the fly and the flower (Fig. 2) because the cost rates $\left(\lambda_{1}\right.$ and $\left.\lambda_{2}\right)$ and other model parameters (the zone enclosed by black lines in Fig. $4 \mathrm{~A}$ and $\mathrm{B}$ ) showed no visible effect on the bias in trait matching.

The negative correlation between the evolutionarily stable length of traits and the cost rates (insets of Fig. 6) suggests that environmental factors (e.g., temperature and wind speed) that could potentially lead to different levels of cost rates will result in the geographical variation of matched traits. Model results highlighted the importance of an imbalance in the cost of trait escalation and weak assortative interactions as two crucial factors for diverse evolutionary outcomes, including sympatric divergence (Figs. 4A and B, 5A and B). The individual-based simulation verified the model predictions (Fig. 5), especially the occurrence of divergent evolution in floral tubes (Fig. 5B). Reciprocal selection first pushed the proboscis and floral tube in a population toward the singular point (evolutionary attractor); at this point divergent selection drove the evolution of polymorphism in flower tubes, and the proboscises of pollinators followed the long-tubed flowers after the branching, consistent with the model prediction and observation (see inset of Figs. 1, 5B). The two subpopulations of the plant from the evolutionary branching, against reality, continued to evolve toward extreme values, suggesting that models of adaptive dynamics have little power in predicting the dynamics far after the branching event (see Discussion for possible reasons).

\section{Discussion}

The hypothesis of coevolution was first proposed by Darwin $(1859,1862)$ to explain how the mutualistic interaction between pollinators and flowers can lead to the development of extraordinarily long proboscises and nectar tubes. Our observation that the benefits to $M$. longirostris and $L$. anceps depend on the trait difference ( $x-y$, proboscis minus floral tube length) supports Darwin's mechanism of reciprocal selection as a plausible model, at least in this system (Fig. 2; Pauw et al. 2009). Working from this basis, we modeled the coevolutionary interaction between plant and pollinator, and additionally explored how the benefits of trait elongation would interact with the costs originally proposed by Wallace (1867). Our model successfully produced two commonly observed patterns in the interaction between coevolving species in nature, that is, geographical variation and bias in trait matching (Steiner and Whitehead 1990; Anderson and Johnson 2008). In addition, the model provides an possible explanation for the enigma of bimodality in floral tube length observed in some populations (Fig. 1; Wasserthal 1997; Pauw et al. 2009; Anderson et al. 2010). The origin of bimodality is particularly interesting because it conceivably represent an early stage in sympatric speciation (Barluenga et al. 2006; Savolainen et al. 2006).

Although this model is designed for this specific pollination system, it can be applied to any coevolutionary systems if the benefit and cost function of interacting traits can be specified. As presented here, the model applies to mutualistic interactions, which have two distinct benefit functions, one for each interacting species, for example, one describing how pollen receipt varies with the trait difference and one describing how nectar receipt varies with the trait difference. However, the model can easily be simplified to become applicable to a wide range of antagonistic relationships, such as those in which defensive and offensive traits coevolve (Thompson and Cunningham 2002; Benkman et al. 2003; Toju and Sota 2006; Decaestecker et al. 2007; Hanifin et al. 2008; King et al. 2011). In such antagonistic relationships, the benefit derived by the defensive species is successful defense and the benefit derived by the offensive species is successful attack, with the probability of successful defense $=1-$ propability of successful attack (Toju and Sota 2006; Nuismer et al. 2007).

An important limitation of models of adaptive dynamics lies in the assumption of asexuality. Although model results can apply well to sexual populations if traits retain a unimodal distribution during evolution (Kisdi and Geritz 1999; Waxman and Gavrilets 2005), evolutionary branching will apply less well to sexual populations because gene flow between morphs can prevent phenotypic divergence (Geritz et al. 1998; Doebeli and Dieckmann 2000; Geritz and Kisdi 2000; Waxman and Gavrilets 2005). Evolutionary branching in sexual populations can only occur if there is assortative mating (or reproductive isolation), that is, there must be a bias in favor of intramorph mating (Dieckmann and Doebeli 1999; Doebeli and Dieckmann 2000). Our individualbased simulation of coevolution in sexual populations (Appendix S7) supports the idea. This is in accordance with the more general observation that speciation requires both disruptive selection and the origin of reproductive isolation (Campbell 2004). Differences in tube length could directly bring about reproductive isolation if short- and long-tubed flowers attach their pollen to discrete parts of the same pollinator (Waterman et al. 2011), or if pollinators exhibit foraging constancy, that is, make fewer than expected intermorph transition while foraging (Grant 1994). Alternatively, postpollination reproductive barriers between morphs might evolve. Our work in progress provides evidence for all three of these modes of reproductive isolation between morphs in a bimodal population of $L$. anceps. Interestingly, this work also provides evidence for lower fitness (seed set) in rare intermediate individuals, a finding consistent with current model predictions. Reproductive barriers between long- and short-tubed morphs and low fitness of the intermediate morph will limit the occurrence of intermediate individuals and might allow divergence to proceed. 
The model successfully shows how geographical variation in the cost of trait elongation translates into geographical variation in the degree of escalation of matching traits (the insets of Fig. 6). Geographical variation in the degree of escalation of coevolved traits is commonly observed (Thompson 2005; Toju and Sota 2006; Anderson and Johnson 2008; Pauw et al. 2009). Indeed, studies have shown that proboscis length is negatively correlated with altitude (Anderson and Johnson 2008) and latitude (Pauw et al. 2009). The cost rates to trait elongation $\left(\lambda_{i}\right)$ could be positively correlated with these geographical variables (which themselves might be proxies for temperature and wind speed). Moreover, the two interactive species could respond differently to the environmental variation, leading to imbalanced costs between the coevolving traits that trigger the divergent selection and the trait polymorphism in the high-cost species (Figs. 4B, 5B). The trade-off between chasing benefit and avoiding cost can be considered a possible mechanism for origin of the bimodal distribution of traits in some populations (e.g., Wasserthal 1997; Pauw et al. 2009). The model highlights the need to explicitly quantify geographical variation in environmental factors and how they affect the fitness of coevolving species via eco-physiological processes (Toju 2008, 2011). Generally, coevolutionary studies emphasize the benefits of trait escalation, but neglect the costs. We also lacked detailed information on costs in our system, but the insensitivity of the model to the form of the cost function (Appendix S1) and the benefit function (Appendix S5) suggests that our model presents the coevolutionary race in a robust manner.

The geographic mosaic theory of coevolution argues that trait mismatching within interacting populations results from trait remixing among geographically differentiated populations via gene flow. Our model provides an alternative, although not mutually exclusive, hypothesis that does not invoke multiple geographically structured populations: when the coevolving species experience asymmetrical costs, Red Queen dynamics (evolutionary cycling) is one of the outcomes which is theoretically possible (Figs. 4A and B, 5C and D). Snapshots of these Red Queen dynamics show significantly mismatched traits. Using models based on population genetics, Nuismer et al. (2010) have also demonstrated that reciprocal selection can lead to a low covariance between interactive traits and thus does not necessarily entail correlations between these traits among multiple populations connected by gene flow. Our results are consistent with Nuismer et al.'s (2010) conclusion but different in that the mismatching of the coevolving traits can also occur within one population due to evolutionary cycling. Although previous studies have shown that coevolution between antagonistic species, such as between predators and prey, can cause Red Queen dynamics (Dieckmann et al. 1995; Doebeli and Dieckmann 2000; Nuismer et al. 2007), we show here that mutualistic interactions can also lead to evolutionary cycling. This suggests that, despite the intuitive difference between antagonistic and mutualistic interactions, they both affect the benefit in a coevolutionary race via trait difference and thus lead to the mathematical consistency of the models for both types of interactions and an equal rich variety of evolutionary dynamics in Darwin's race (Nuismer et al. 2007).

The model reveals the importance of pollinator preference for plants with tube lengths similar to their proboscis length as the key for releasing species from evolutionary traps and giving rise to evolutionary branching. Without these assortative interactions between partners, reciprocal selection can drive the higher cost species to a phenotype with minimum fitness but not followed by an evolutionary branching, in contrast to the classic theory of adaptive dynamics (Doebeli and Dieckmann 2000). Random interactions cannot maintain polymorphism because individuals with higher fitness traits can exclude individuals with other traits immediately after the evolutionary branching, that is, two branches cannot coexist (Fig. 5A). On the contrary, even very weak assortative interaction can lead to frequencydependent selection in the population capable of maintaining bimodality (Doebeli and Dieckmann 2000). Few, if any, empirical studies have looked for assortative interactions of this type and our data (Fig. 3) offers only very limited support. Progress in optimal foraging theory, however, provides interesting clues as to why assortative interactions play such a key role in unlocking the evolutionary trap. First, optimal foraging theory can directly give rise to assortative interactions between floral tubes and pollinator proboscis when there is obvious interspecific resource competition (Rodríguez-Gironés and Santamaría 2007); in our case, intermorph competition for pollen receipt. Second, optimal foraging theory can further explain how two competing morphs can coexist by virtue of differential resource access and utilization (Richards et al. 2000), thus unlocking the evolutionary trap and allowing the coexistence of the two morphs after the evolutionary branching (Richards et al. 2000). A nonlinear functional response derived from optimal foraging can also lead to preferential (nonrandom) interactions in pollination networks (Zhang et al. 2011). Exploring the relationship between optimal foraging in pollinators and assortative mating in plants will clearly be a fruitful avenue for both empirical and theoretical studies

The model presented here has demonstrated that Darwin's coevolutionary race, if subjected to a constraint of cost to trait escalation and weak assortative interaction, can produce the three observed patterns in coevolving pollination systems: geographical variation of traits, bias in trait matching, and polymorphism within populations. However, this does not necessarily imply that other alternative processes cannot produce these patterns. For instance, the pollinator shift hypothesis of one-sided selection has been suggested as an alternative process for trait escalation in some systems (Wasserthal 1997; Whittall and Hodges 2007); 
a similar adaptive-dynamics model assuming one-sided selection, instead of Darwin's reciprocal selection, also produced partially accordant results except for the direction of the bias in trait matching (Appendix S10). The weak discrimination power of our model results from the lack of data for parameterizing the cost function. Moreover, to reduce the model complexity, other factors that could be important in the evolution of pollination systems have been neglected, for example, population dynamics (Holland et al. 2002), density dependence (Rymer et al. 2010), nectar variation (Manning and Goldblatt 1997; Wasserthal 1997; Rymer et al. 2010), pollinator behavior (Richards et al. 2000; Rymer et al. 2010), and gene flow (Nuismer et al. 1999; Thompson 2005). In addition, our model only considered a one-to-one pollination system, whereas the reality could well be a pollination network (Bascompte et al. 2003; Rodríguez-Gironés and Santamaría 2007; RodríguezGironés and Llandres 2008; Zhang et al. 2011), given that other long-tubed plant species do exist in our sites at low density (Pauw et al. 2009). Considering multiple pollinators and plant species in a network and various factors listed above could potentially increase the nonlinearity of the system and restrain the two subpopulations from evolving toward extremes after branching (Fig. 5B), but such exhaustive models have yet to be developed. However, our model can already produce such a rich variety of evolutionary behaviors; adding these factors, although could lead to more realistic predictions, will inevitably complicate the calculation. The parsimony rule of building simpler models should be favored.

In conclusion, the model presented here provides a simple and mechanistic understanding of how Darwin's coevolutionary race between flowers and their pollinators can give rise to polymorphism in interacting populations. The model highlights three important gaps in the empirical data that are currently available for coevolving systems and thus reveals new avenues of empirical studies. First, the model reveals that an imbalance in the benefit and cost experienced by one species relative to the other could lead to the observed bias in trait matching and the bimodal distribution of the trait in the high-cost species. Clearly, we need to attempt to quantify the cost to trait escalation and how it varies across the landscape. Second, the model predicts that bimodality will only manifest when interactions are assortative, that is, short proboscid pollinators prefer to forage from short-tubed flowers, whereas long proboscid individuals prefer long-tubed flowers. This is another area where field data are needed. Finally, the model suggests that trait mismatching can result from the evolutionary cycling of traits (i.e., Red Queen dynamics), rather than as a result of trait remixing by gene flow as the Geographic Mosaic Theory of Coevolution would predict. We thus need long-term or paleontological data $(>1000$ generations) (e.g., Decaestecker et al. 2007) to test whether evolutionary cycling of traits is occurring in real world situations, especially for the communities with trait mismatching.

\section{ACKNOWLEDGMENTS}

We are grateful to B. Anderson, S. D. Johnson, S. C. H. Barrett, V. Savolainen, and four anonymous referees for constructive comments. This work was supported by the National Research Foundation (NRF) and the DST-NRF Centre of Excellence for Invasion Biology at Stellenbosch University.

\section{LITERATURE CITED}

Alexandersson, R., and S. D. Johnson. 2002. Pollinator-mediated selection on flower-tube length in a hawkmoth-pollinated Gladiolus (Iridaceae). Proc. R. Soc. Lond. Ser. B 269:631-636.

Anderson, B., and S. D. Johnson. 2008. The geographical mosaic of coevolution in a plant-pollinator mutualism. Evolution 62:220-225.

Anderson, B., R. Alexandersson, and S. D. Johnson. 2010. Evolution and coexistence of pollination ecotypes in an African gladiolus (Iridaceae). Evolution 64:960-972.

Barluenga, M., K. N. Stölting, W. Salzburger, M. Muschick, and A. Meyer, 2006. Sympatric speciation in Nicaraguan Crater lake cichlid fish. Nature 439:719-723.

Bascompte, J., P. Jordano, C. J. Melian, and J. M. Olesen. 2003. The nested assembly of plant-animal mutualistic networks. Proc. Natl. Acad. Sci USA 100:9383-9387.

Beaumont, M. A. 2010. Approximate bayesian computation in evolution and ecology. Annu. Rev. Ecol. Evol. Syst. 41:379-406.

Benkman, C. W., T. L. Parchman, A. Favis, and A. M. Siepielski. 2003. Reciprocal selection causes a coevolutionary arms race between crossbills and lodgepole pine. Am. Nat. 162:182-194.

Bergelson, J., G. Dwyer, and J. J. Emerson. 2001. Models and data on plantenemy coevolution. Annu. Rev. Genet. 35:469-499.

Brodie, E. D., and B. J. Ridenhour. 2003. Reciprocal selection at the phenotypic interface of coevolution. Integr. Comp. Biol. 43:408-418.

Campbell, D. R. 2004. Natural selection in Ipomopsis hybrid zones: implications for ecological speciation. New Phytol. 161:83-90.

Darwin, C. 1859. The origin of species by means of natural selection, or the preservation of favoured races in the struggle for life. John Murray, London.

- 1862. On the various contrivances by which British and foreign orchids are fertilized by insect. Murray, London.

Decaestecker, E., S. Gaba, J. A. M. Raeymaekers, R. Stoks, L. Van Kerckhoven, D. Ebert, and L. De Meester. 2007. Host-parasite 'Red Queen' dynamics archived in pond sediment. Nature 450:870-874.

Dercole, F., and S. Rinaldi. 2008. Analysis of evolutionary processes: the adaptive dynamics approach and its applications. Princeton Univ. Press, Princeton.

Dieckmann, U., and M. Doebeli. 1999. On the origin of species by sympatric speciation. Nature 400:354-357.

Dieckmann, U., and R. Law. 1996. The dynamical theory of coevolution: a derivation from stochastic ecological processes. J. Math. Biol. 34:579612.

Dieckmann, U., P. Marrow, and R. Law. 1995. Evolutionary cycling in predator-prey interactions: population dynamics and the Red Queen. J. Theor. Biol. 176:91-102.

Dieckmann, U., M. Doebeli, J. A. J. Metz, and D. Tautz. 2004. Adaptive speciation. Cambridge Univ. Press, Cambridge, U.K.

Doebeli, M., and U. Dieckmann. 2000. Evolutionary branching and sympatric speciation caused by different types of ecological interactions. Am. Nat. 156:S77-S101.

Duffy, M. A., C. E. Brassil, S.R. Hall, A.J. Tessier, C.E. Caceres, and J. K. Conner. 2008. Parasite-mediated disruptive selection in a natural Daphnia population. BMC Evol. Biol. 8:80. 
Ennos, R. A. 2008. Spurred on by pollinators. Heredity 100:3-4.

Geritz, S. A. H., and E. Kisdi. 2000. Adaptive dynamics in diploid, sexual populations and the evolution of reproductive isolation. Proc. R. Soc. B 267:1671-1678.

Geritz, S. A. H., E. Kisdi, G. Meszena, and J. A. J. Metz. 1998. Evolutionarily singular strategies and the adaptive growth and branching of the evolutionary tree. Evol. Ecol. 12:35-57.

Grant, V. 1994. Modes and origins of mechanical and ethological isolation in angiosperms. Proc. Natl. Acad. Sci. USA 91:3-10.

Hanifin, C.T., E.D. Brodie, Jr., and E.D. Brodie, III. 2008. Phenotypic mismatches reveal escape from arms-race coevolution. PLoS Biology 6:e60.

Herrera, C. M. 1993. Selection on floral morphology and environmental determinants of fecundity in a hawkmoth-pollinated violet. Ecol. Monogr. 63:251-275.

Hodges, S. A., and J. B. Whittall. 2008. One-sided evolution or two? A reply to Ennos. Heredity 100:541-542.

Holland, J. N., D. L. DeAngelis, and J. L. Bronstein. 2002. Population dynamics and mutualism: functional responses of benefits and costs. Am. Nat. 159:231-244.

Janzen, D. H. 1980. When is it coevolution? Evolution 34:611-612.

Johnson, S. D., and K. E. Steiner. 1997. Long-tongued fly pollination and evolution of floral spur length in the Disa draconis complex (Orchidaceae). Evolution 51:45-53.

Kiester, A. R., R. Lande, and D. W. Schemske. 1984. Models of coevolution and speciation in plants and their pollinators. Am. Nat. 124: 220-243.

King, K. C., L. F. Delph, J. Jokela, and C. M. Lively. 2011. Coevolutionary hotspots and coldspots for host sex and parasite local adaptation in a snail-trematode interaction. Oikos 120:1335-1340.

Kisdi, E., and S. A. H. Geritz. 1999. Adaptive dynamics in allele space: evolution of genetic polymorphism by small mutations in a heterogeneous environment. Evolution 53:993-1008.

Langmore, N. E., S. Hunt, and R. M. Kilner. 2003. Escalation of a coevolutionary arms race through host rejection of brood parasitic young. Nature 422:157-160.

Little, K. J., G. Dieringer, and M. Romano. 2005. Pollination ecology, genetic diversity and selection on nectar spur length in Platanthera lacera (Orchidaceae). Plant Species Biol. 20:183-190.

Maad, J. 2000. Phenotypic selection in hawkmoth-pollinated Platanthera bifolia: targets and fitness surfaces. Evolution 54:112-123.

Manning, J. C., and P. Goldblatt. 1997. The Moegistorhynchus longirostris (Diptera: Nemestrinidae) pollination guild: long-tubed flowers and a specialized long-proboscid fly pollination system in southern Africa. Plant Syst. Evol. 206:51-69.

Muchhala, N., and J. D. Thomson. 2009. Going to great lengths: selection for long corolla tubes in an extremely specialized bat-flower mutualism. Proc. R. Soc. B 276:2147-2152.

Navarro, L., and R. Medel. 2009. Relationship between floral tube length and nectar robbing in Duranta erecta L. (Verbenaceae). Biol. J. Linn. Soc. 96:392-398.

Nilsson, L. A. 1998. Deep flowers for long tongues. Trends Ecol. Evol. 13:259-260.

Nuismer, S. L., J. N. Thompson, and R. Gomulkiewicz. 1999. Gene flow and geographically structured coevolution. Proc. R. Soc. Lond. Ser. B 266:605-609.
Nuismer, S. L., B. J. Ridenhour, and B. P. Oswald. 2007. Antagonistic coevolution mediated by phenotypic difference between quantitative traits. Evolution 61:1823-1834.

Nuismer, S. L., R. Gomulkiewicz, and B. J. Ridenhour. 2010. When is correlation coevolution? Am. Nat. 175:525-537.

Pauw, A., J. Stofberg, and R. J. Waterman. 2009. Flies and flowers in Darwin's race. Evolution 63:268-279.

Richards, S. A., R. M. Nisbet, W. G. Wilson, and H. P. Possingham. 2000. Grazers and diggers: exploitation competition and coexistence among foragers with different feeding strategies on a single resource. Am. Nat. 155:266-279.

Rodríguez-Gironés, M. A., and A. L. Llandres. 2008. Resource competition triggers the co-evolution of long tongues and deep corolla tubes. PLoS One 3:e2992.

Rodríguez-Gironés, M. A., and L. Santamaría. 2007. Resource competition, character displacement, and the evolution of deep corolla tubes. Am. Nat. 170:455-464.

Rymer, P. D., S. D. Johnson, and V. Savolainen. 2010. Pollinator behaviour and plant speciation: can assortative mating and disruptive selection maintain distinct floral morphs in sympatry? New Phytol. 188:426-436.

Savolainen, V., M. C. Anstett, C. Lexer, I. Hutton, J. J. Clarkson, M. V. Norup, M. P. Powell, D. Springate, N. Salamin, and W. J. Baker. 2006. Sympatric speciation in palms on an oceanic island. Nature 441: 210-213.

Steiner, K. E., and V. B. Whitehead. 1990. Pollinator adaptation to oil-secreting flowers-Rediviva and Diascia. Evolution 44:1701-1707.

Thompson, J. N. 2005. The geographic mosaic of coevolution. Chicago Univ. Press, Chicago.

Thompson, J. N., and Cunningham, B.M. 2002. Geographic structure and dynamics of coevolutionary selection. Nature 417: 735-738.

Toju, H. 2008. Fine-scale local adaptation of weevil mouthpart length and camellia pericarp thickness: altitudinal gradient of a putative arms race. Evolution 62:1086-1102. . 2011. Weevils and camellias in a Darwin's race: model system for the study of eco-evolutionary interactions between species. Ecol. Res. 26:239-251

Toju, H., and T. Sota. 2006. Imbalance of predator and prey armament: geographic clines in phenotypic interface and natural selection. Am. Nat. 167:105-117.

Wallace, A. R. 1867. Creation by law. Q. J. Sci. 4:470-488.

Wasserthal, L. T. 1997. The pollinators of the Malagasy star orchids Angraecum sesquipedale, A-sororium and A-compactum and the evolution of extremely long spurs by pollinator shift. Bot. Acta 110:343-359.

1998. Deep flowers for long tongues. Trends Ecol. Evol. 13:459-460.

Waterman, R. J., M. I. Bidartondo, J. Stofberg, J. K. Combs, G. Gebauer, V. Savolainen, T. G. Barraclough, and A. Pauw. 2011. The Effects of above- and belowground mutualisms on orchid speciation and coexistence. Am. Nat. 177:E54-E68.

Waxman, D., and S. Gavrilets. 2005. 20 questions on adaptive dynamics. J. Evol. Biol. 18:1139-1154.

Whittall, J. B., and S. A. Hodges. 2007. Pollinator shifts drive increasingly long nectar spurs in columbine flowers. Nature 447:U706-U712.

Zhang, F., C. Hui, and J. S. Terblanche. 2011. An interaction switch predicts the nested architecture of mutualistic networks. Ecol. Lett. 14:797-803.

Associate Editor: V. Savolainen 


\section{Supporting Information}

Additional Supporting Information may be found in the online version of this article at the publisher's web site:

Appendix S1. The effect of alterative cost functions on the evolutionary outcomes.

Appendix S2. A comparison of assortative interactions with Gaussian versus Logistic forms.

Appendix S3. Analysis of the adaptive dynamics.

Appendix S4. Sensitivity to the pollination probability by a single pollen grain.

Appendix S5. Sensitivity to the parameters in the benefit function.

Appendix S6. The effect of the mutation rate and variance on the evolutionary branching.

Appendix S7. A simulation of coevolution in sexual populations.

Appendix S8. Experimental observations of the trait lengths in the fly-flower pollination system.

Appendix S9. Multi equilibria in the fly-flower coevolution without assortative interactions.

Appendix S10. Analysis of a one-sided selection model (the pollinator shift hypothesis). 